\title{
The characterization of a line arrangement whose fundamental group of the complement is a direct sum of free groups
}

\author{
Meital Eliyahu \\ ERAN LIBERMAN \\ MALKA SCHAPS \\ MINA TEICHER
}

\begin{abstract}
Kwai Man Fan proved that if the intersection lattice of a line arrangement does not contain a cycle, then the fundamental group of its complement is a direct sum of infinite and cyclic free groups. He also conjectured that the converse is true as well. The main purpose of this paper is to prove this conjecture.
\end{abstract}

14F35, 32S22; 14N30, 52C30

\section{Introduction}

An arrangement of lines is a finite collection of $\mathbb{C}$-affine subspaces of dimension 1 . For such an arrangement $\Sigma \subseteq \mathbb{C}^{2}$, there is a natural projective arrangement $\Sigma^{*}$ of lines in $\mathbb{C} \mathbb{P}^{2}$ associated to it, obtained by adding to each line its corresponding point at infinity. The problem of finding connections between the topology of $\mathbb{C}^{2}-\Sigma$ and the combinatorial theory of $\Sigma$ is one of the main problems in the theory of line arrangements (see for example Cohen and Suciu [3]). The main motivations for studying the topology of $\mathbb{C}^{2}-\Sigma$ are derived from the areas of hypergeometric functions, singularity theory and algebraic geometry.

Given an arrangement $\Sigma$, we define the graph $G(\Sigma)$ in the way it is defined by Fan [7]. We first connect all higher multiple points of $\Sigma$ which lie on a line $L_{i}$ by an arbitrary simple arc $\alpha_{i} \subset L_{i}$ which does not go through any double point of $\Sigma$. Taking the union of the set of all higher multiple points and all of this simple arcs, we obtain $G(\Sigma)$. Note that if 3 points are on the same line we do not consider it as a cycle.

In 1994, Jiang and Yau [11] defined the concept of a "nice" arrangement. For $\Sigma$, they define a graph $G(V, E)$ : The vertices are the multiple points of $\Sigma$, and vertices $u, v$ are connected by an edge if there exists $l \in \Sigma$ such that $u, v \in l$. For $v \in V$ define a 
subgraph $G_{\Sigma}(v)$ : The vertex set is $v$ and all his neighbors from $\Sigma$ (note this definition differs from the definition of Fan).

An arrangement $\Sigma$ is nice if there is $V^{\prime} \subset V$ such that $E_{\Sigma}(v) \cap E_{\Sigma}(u)=\varnothing$ for all $u, v \in V^{\prime}$, and if we delete the vertex $v$ and the edges of its subgraph $G_{\Sigma}(v)$ from $G$, for all $v \in V^{\prime}$ we get a forest (ie a graph without cycles).

Jiang and Yau have proven several properties of nice arrangements:

(a) If $A_{1}, A_{2}$ are nice arrangements and their lattices are isomorphic, then their complements $\mathbb{C}^{2}-A_{1}$ and $\mathbb{C}^{2}-A_{2}$ are diffeomorphic. This property naturally implies that $\pi_{1}\left(\mathbb{C}^{2}-A_{1}\right) \cong \pi_{1}\left(\mathbb{C}^{2}-A_{2}\right)$.

(b) As a consequence of (a), they showed that the presentation of the fundamental group of the complement can be written explicitly and depends only on the lattice of the line arrangement.

In 2005, Wang and Yau [20] continued in this direction and proved that the results of Jiang and Yau hold for a much larger family of arrangements, which they call simple arrangements.

Falk [4] finds several examples of line arrangements with the same homotopy types but with different lattices. Fan [6] proved that for up to 6 lines, the fundamental group of a real line arrangement is determined by the lattice. Garber, Teicher and Vishne [9] proved this for a real line arrangement with up to 8 lines.

Let $G, H$ be groups whose abelianizations are free abelian groups of finite rank. Choudary, Dimca and Papadima [2] defined a set $\Phi$ of natural group isomorphisms $\phi: G \rightarrow H$ (which they call a 1-marking). In the case of $G=\pi_{1}\left(\mathbb{C}^{2}-\Sigma\right.$ ) (where $\Sigma$ is an affine arrangement), $\phi$ takes the topological structure of $\mathbb{C}^{2}-\Sigma$ into consideration. They prove that if $A, B$ are line arrangements and $\mathrm{B}$ is nice in the sense of Jiang and Yau, then the lattices are isomorphic if and only if there is an isomorphism $\phi \in \Phi$ where $\phi: \pi_{1}\left(\mathbb{C}^{2}-A\right) \stackrel{\sim}{\rightarrow} \pi_{1}\left(\mathbb{C}^{2}-B\right)$.

Fan [7] showed that if the graph $G(\Sigma)$ is a forest (ie a graph without cycles), then the fundamental group is a direct sum of free groups. He also conjectured that the converse of his theorem is true. In [5] Fan proved that if the fundamental group of the complement is a direct sum of free groups, then the arrangement is composed of parallel lines.

In this paper, we prove his conjecture. Our theorem will state that if the fundamental group is isomorphic to a direct sum of free groups, then the graph has no cycles. We would like to emphasize that we make no restrictions on our isomorphisms. 
The structure of the paper is as follows. In Section 2, we give basic definitions related to groups. In Section 3 we give basic definitions related to line arrangements and the fundamental group of the complement of a line arrangement. In Section 4, we define a function and a special set induced by it. Section 5 deals with some special properties of fundamental groups of the complements of line arrangements which are direct sum of free groups. In Section 6, we prove the main result of the paper.

\section{Definitions and notation}

This section presents the needed definitions for the paper.

\subsection{Lower central series}

We start by defining the lower central series of a group $G$ which will be used throughout the paper.

Definition 2.1 (Commutator group and lower central series) Let $G$ be a group. The commutator group of $G$ is

$$
G^{\prime}=G_{2}=[G, G]=\left\langle a b a^{-1} b^{-1} \mid a, b \in G\right\rangle
$$

The subgroup $G^{\prime}$ is normal in $G$ with an abelian quotient. We can define the lower central series of $G$ recursively:

$$
\begin{aligned}
G_{1} & =G \\
G_{2} & =[G, G] \\
G_{3} & =\left[G, G_{2}\right] \\
& \vdots \\
G_{n} & =\left[G, G_{n-1}\right] .
\end{aligned}
$$

Since $G_{n+1}$ contains the commutators of $G_{n}$, we have $G_{n+1} \triangleleft G_{n}$ and the quotient $G_{n} / G_{n+1}$ is abelian for all $n \in \mathbb{N}$.

To understand these groups, the following identities are needed.

Proposition 2.2 (Witt-Hall identities [15])

(1) $[a, b][b, a]=e$.

(2) $[a, b c]=[a, b][a, c][[c, a], b]$.

(3) $[a b, c]=[a,[b, c]][b, c][a, c]$. 
From the second and third identities we get:

Lemma 2.3 Let $G$ be a group and let $\left\{x_{1}, \ldots, x_{k}\right\}$ be the generators of $G$. Then

$$
G_{2} / G_{3}=\left\langle\left[x_{i}, x_{j}\right] \mid i \neq j, 1 \leq i, j \leq k\right\rangle .
$$

Proof If $[x y, z] \in G_{2}$, then

$$
[x y, z]=x y z(x y)^{-1} z^{-1}=x y z y^{-1} x^{-1} z^{-1}=x[y, z] x^{-1}[x, z] .
$$

Over $G_{3}$, we have $[x,[y, z]]=x[y, z] x^{-1}[y, z]^{-1}=e \Rightarrow[y, z]=x[y, z] x^{-1}$. We get

$$
[x y, z]=[y, z][x, z] \text { over } G_{3} .
$$

This result means that $G_{2} / G_{3}$ is finitely generated by the set

$$
\left\{\left[x_{i}, x_{j}\right] \mid i \neq j, 1 \leq i, j \leq k\right\},
$$

where $x_{1}, \ldots, x_{k}$ are the generators of $G$.

Proposition 2.4 Let $G=\left\langle x_{1}, \ldots, x_{k} \mid R\right\rangle$, where the relations $R$ are commutator type relations (ie every relation $r \in R$ can be written as $r=\left[w_{1}, w_{2}\right], w_{1}, w_{2} \in G$ ). Then

$$
G_{2} / G_{3}=\left\langle\left[x_{i}, x_{j}\right] \mid \begin{array}{l}
{\left[x_{k}, x_{l}\right]=\left[x_{l}, x_{k}\right]^{-1},} \\
\text { all generators commute, } \\
R^{*}
\end{array}\right\rangle
$$

where $R^{*}$ is set of the relations $R$ written by means of the generators of $G_{2}$, taken modulo $G_{3}$.

The next Definition and theorem will give us a better understanding of $G_{2} / G_{3}$ and help us in the future.

Definition 2.5 [10, page 165] Let $G$ be a group generated by $x_{1}, \ldots, x_{r}$. We consider formal words or strings $b_{1} \cdot b_{2} \cdots b_{n}$ where each $b$ is one of the generators. We also introduce formal commutators $c_{j}$ and weights $\omega\left(c_{j}\right)$ by the rules:

(1) $c_{i}=x_{i}, i=1, \ldots, r$ are the commutators of weight 1 ; ie $\omega\left(x_{i}\right)=1$.

(2) If $c_{i}$ and $c_{j}$ are commutators, then $c_{k}=\left[c_{i}, c_{j}\right]$ and $\omega\left(c_{k}\right)=\omega\left(c_{i}\right)+\omega\left(c_{j}\right)$.

Theorem 2.6 (Basis theorem [10]) If $F$ is the free group with free generators $y_{1}, \ldots, y_{r}$ and if in a sequence of basic commutators $c_{1}, \ldots, c_{t}$ are those of weights $1,2, \ldots, n$ then an arbitrary element $f \in F$ has a unique representation $f=c_{1}^{e_{1}} c_{2}^{e_{2}} \cdots c_{t}^{e_{t}}$ $\bmod F_{n+1}$.

The basic commutators of weight $n$ form a basis for the free abelian group $F_{n} / F_{n+1}$. 


\subsection{Line arrangements}

Definition 2.7 (Line arrangement) A line arrangement $\Sigma=\left\{L_{1}, \ldots, L_{s}\right\} \subseteq \mathbb{C}^{2}$ is a union of copies of $\mathbb{C}^{1}$.

Remark 2.8 Each time we are mentioning a line arrangement $\Sigma \subseteq \mathbb{C}^{2}$, we assume that there are no parallel lines in $\Sigma$. Pay attention we can project every line arrangement in $\mathbb{C P}^{2}$ to $\mathbb{C}^{2}$ such that the new arrangement does not contain parallel lines.

Definition 2.9 Let $\Sigma$ be a line arrangement. An intersection point in $\Sigma$ is called simple if there are precisely two lines which meet at that point. Otherwise, we call it multiple.

Definition 2.10 (Cycle) A cycle is a nonempty ordered set of multiple intersection points $\left\{p_{1}, \ldots, p_{k}\right\}$, such that any pair of adjacent points $p_{j}, p_{j+1}$ and the points $p_{1}, p_{k}$ are connected by lines of the arrangement. Moreover, if $1 \leq j \leq k-2$, then the line connecting $p_{j}$ to $p_{j+1}$ and the line connecting $p_{j+1}$ to $p_{j+2}$ are different. Also, the line connecting $p_{k-1}$ to $p_{k}$ is different from the line connecting $p_{k}$ to $p_{1}$.

Let $\Sigma \subseteq \mathbb{C P}^{2}$ be a line arrangement. The invariant $\beta(\Sigma)$ which is defined in [7], counts the number of independent cycles in the graph.

Fan [7] showed that $\beta(\Sigma)=0$ if and only if $G(\Sigma)$ has no cycles.

In an analogous way for $\Sigma \subseteq \mathbb{C}^{2}$, we define $\beta(\Sigma):=\beta\left(\Sigma \cup L_{\infty}\right)$ where $L_{\infty}$ is the projective line at infinity of $\mathbb{C}^{2}$ and $G(\Sigma):=G\left(\Sigma \cup L_{\infty}\right)$. Note that the new definitions are well defined.

One of the most important invariants of a line arrangement is the fundamental group of its complement, denoted by $\pi_{1}\left(\mathbb{C}^{2}-\Sigma\right)$.

The next lemma presents its computation.

Lemma 2.11 (Constructing the fundamental group [1; 18; 19; 3, page 304]) Let $\Sigma=\left\{L_{1}, \ldots, L_{n}\right\} \subseteq \mathbb{C}^{2}$ be a line arrangement that is enumerated as in [3].

We associate a generator $\Gamma_{i}$ to each line $L_{i}$ with base point as in [3] such that

$$
G=\pi_{1}\left(\mathbb{C}^{2}-\Sigma\right)=\left\langle\Gamma_{1}, \ldots, \Gamma_{n} \mid R\right\rangle,
$$

where $R$ is a set of relations generated as follows.

Every intersection point of lines $L_{i_{1}}, \ldots, L_{i_{m}}$ creates a set of relations

$$
\Gamma_{i_{1}}^{x_{1}} \Gamma_{i_{2}}^{x_{2}} \cdots \Gamma_{i_{m}}^{x_{m}}=\Gamma_{i_{m}}^{x_{m}} \Gamma_{i_{1}}^{x_{1}} \cdots \Gamma_{i_{m-1}}^{x_{m-1}}=\Gamma_{i_{2}}^{x_{2}} \cdots \Gamma_{i_{m}}^{x_{m}} \Gamma_{i_{1}}^{x_{1}}
$$

where $x_{i} \in G$ and $\Gamma_{i}^{x_{i}}=x_{i}^{-1} \Gamma_{i} x_{i}$. 
It is easy to see that this set is equivalent to the following set:

$$
\left[\Gamma_{i_{j}}^{x_{j}}, \Gamma_{i_{1}}^{x_{1}} \cdots \Gamma_{i_{m}}^{x_{m}}\right]=e, \quad 1 \leq j \leq m .
$$

We get a similar presentation for a real arrangement by using the Moishezon-Teicher algorithm [16] and the van Kampen Theorem [13].

Notation 2.12 We denote by $\mathcal{P}$ the set of intersection points. For $p \in \mathcal{P}$, we denote by $\Gamma(p)$ the set of generators attached to the lines passing through the point $p$ and by $\Gamma(p)^{c}$ the set of generators attached to the lines not passing through the point $p$.

\section{Decomposition of $G_{2} / G_{3}$}

Let $G$ be a group. The abelianization of $G$, denoted by $\bar{G}$, is $\bar{G}=G / G_{2}$. If $g \in G$, we denote $\bar{g}=g \cdot G_{2}$.

Remark 3.1 Let $\Sigma \in \mathbb{C}^{2}$ be a line arrangement, and let $p \in \mathcal{P}$. Then, $G=$ $\pi_{1}\left(\mathbb{C}^{2}-\Sigma\right)=\left\langle\Gamma(p), \Gamma(p)^{c} \mid R\right\rangle$,

$$
G / G_{2}=\bar{G}=\operatorname{Ab}(G)=\left\langle\overline{\Gamma(p)}, \overline{\Gamma(p)^{c}} \mid R,[x, y]=e, x, y \in \Gamma(p) \cup \Gamma(p)^{c}\right\rangle .
$$

This Lemma is an immediate implementation of the last section.

Lemma 3.2 (An implementation for line arrangements) Let $\Sigma$ be a line arrangement, then the abelian group $G_{2} / G_{3}$ can be written as

$$
G_{2} / G_{3}=\left\langle\begin{array}{l|l}
{\left[\Gamma_{i}, \Gamma_{j}\right]} & \begin{array}{l}
{\left[\Gamma_{i}, \Gamma_{j}\right]=\left[\Gamma_{j}, \Gamma_{i}\right]^{-1},} \\
\left.\left[\Gamma_{i}\right], \Gamma_{j}\right]\left[\Gamma_{k}, \Gamma_{l}\right]=\left[\Gamma_{k}, \Gamma_{l}\right]\left[\Gamma_{i}, \Gamma_{j}\right], \\
\prod_{\Gamma_{x} \in \Gamma(p)}\left[\Gamma_{x}, \Gamma_{y}\right], \quad p \in \mathcal{P}, \Gamma_{y} \in \Gamma(p)
\end{array}
\end{array}\right\}
$$

Proof A simple implementation of Proposition 2.4 on the presentation of $G$ from Lemma 2.11.

Remark 3.3 We can see that if $\Gamma_{1}$ and $\Gamma_{2}$ are associated with lines meeting in one point and $\Gamma_{3}$ and $\Gamma_{4}$ are associated with lines meeting in a different point, there is no relation combining $\left[\Gamma_{1}, \Gamma_{2}\right]$ and $\left[\Gamma_{3}, \Gamma_{4}\right]$. Therefore,

$$
G_{2} / G_{3}=\bigoplus_{p \in \mathcal{P}} C_{p}
$$


where

$\left.C_{p}=\left\langle\Gamma_{i}, \Gamma_{j}\right], \Gamma_{i}, \Gamma_{j} \in \Gamma(p) \mid \begin{array}{l}{\left[\Gamma_{i}, \Gamma_{j}\right]=\left[\Gamma_{j}, \Gamma_{i}\right]^{-1},} \\ {\left[\Gamma_{i}, \Gamma_{j}\right]\left[\Gamma_{k}, \Gamma_{l}\right]=\left[\Gamma_{k}, \Gamma_{l}\right]\left[\Gamma_{i}, \Gamma_{j}\right], \Gamma_{i}, \Gamma_{j}, \Gamma_{k}, \Gamma_{l} \in \Gamma(p),} \\ \prod_{\Gamma_{x} \in \Gamma(p)}\left[\Gamma_{x}, \Gamma_{y}\right], \Gamma_{y} \in \Gamma(p)\end{array}\right\}$.

We can see that the generators of the different groups $C_{p}$ in the direct sum are disjoint. Consequently, let $x \in G_{2} / G_{3}$, then $x=\bigoplus_{p \in \mathcal{P}} c_{p}$, where $c_{p} \in C_{p}$.

For each $r \in \mathcal{P}$, consider the projection

$$
\xi_{r}: G_{2} / G_{3} \rightarrow C_{r}
$$

given by

$$
\xi_{r}(x)=\xi_{r}\left(\bigoplus_{p \in \mathcal{P}} c_{p}\right)=c_{r} .
$$

If $\Gamma_{i} \in \Gamma(r)^{c}$, then for all $\Gamma_{j}, \xi_{r}\left(\left[\Gamma_{i}, \Gamma_{j}\right]\right)=\xi_{r}\left(\left[\Gamma_{j}, \Gamma_{i}\right]\right)=e$. If $\Gamma_{i}, \Gamma_{j} \in \Gamma(r)$, then $\xi_{r}\left(\left[\Gamma_{i}, \Gamma_{j}\right]\right)=\left[\Gamma_{i}, \Gamma_{j}\right]$.

\section{The stabilizer of an intersection point}

Let $G=\pi_{1}\left(\mathbb{C}^{2}-\Sigma\right)$. Define

$$
\begin{gathered}
f: G / G_{2} \times G / G_{2} \longrightarrow G_{2} / G_{3} \\
f(\bar{a}, \bar{b})=[a, b] / G_{3} .
\end{gathered}
$$

by

This function is well-defined: If $\overline{a_{1}}=\overline{a_{2}}$ and $\overline{b_{1}}=\overline{b_{2}}$, then $a_{2}=a_{1} x$ where $x \in G_{2}$, $b_{2}=b_{1} y$ where $y \in G_{2}$. Then, by Proposition 2.2,

$$
\left[a_{2}, b_{2}\right]=\left[a_{1} x, b_{1} y\right]=\left[a_{1} x, b_{1}\right]\left[a_{1} x, y\right]=\left[a_{1} x, b_{1}\right]=\left[a_{1}, b_{1}\right]\left[x, b_{1}\right]=\left[a_{1}, b_{1}\right],
$$

so $f\left(\overline{a_{1}}, \overline{b_{1}}\right)=f\left(\overline{a_{2}}, \overline{b_{2}}\right)$.

The following lemma presents some properties of $f$ :

Lemma 4.1 Let $a, b, c \in G / G_{2}$. Then:
(1) $f(a \cdot b, c)=f(a, c) \cdot f(b, c)$.
(2) $f(a, b \cdot c)=f(a, b) \cdot f(a, c)$.
(3) $f\left(a^{n}, b^{m}\right)=f(a, b)^{n m}$ for $m, n \in \mathbb{Z}$.
(4) $f(b, a)=(f(a, b))^{-1}$. 
Proof (1) Let $A, B, C \in G$ such that $\bar{A}=a, \bar{B}=b$, and $\bar{C}=c$. This means that $\overline{A B}=a b$, so by definition, $f(a b, c)=[A B, C] / G_{3}=([A, C][B, C]) / G_{3}$ which by definition is equal to $f(a, c) f(b, c)$.

(2) The proof is the same as (1).

(3) Simple induction on (1) and (2).

(4) Let $A, B \in G$ such that $\bar{A}=a, \bar{B}=b$. By definition,

$$
f(b, a)=[B, A] / G_{3}=\left([A, B] / G_{3}\right)^{-1}=(f(a, b))^{-1} .
$$

Now for any $\bar{x} \in G / G_{2}$ we define

$$
S(\bar{x})=\left\{y \in G / G_{2} \mid f(\bar{y}, \bar{x})=e_{G_{3}}\right\} .
$$

By Lemma 4.1, $S(\bar{x})$ is a subgroup of $G / G_{2}$.

From now on, we talk about a specific intersection point $Q$. The lines passing through this point are $\left\{L_{i_{1}}, \ldots, L_{i_{m}}\right\}$. Define $M=\Gamma_{i_{1}}^{x_{1}} \Gamma_{i_{2}}^{x_{2}} \cdots \Gamma_{i_{m}}^{x_{m}}$. Then, as noted in Lemma 2.11 , the relations induced from the point $\mathrm{Q}$ can be translated to $\left[\Gamma_{i_{1}}^{x_{1}}, M\right]=\cdots=$ $\left[\Gamma_{i_{m}}^{x_{m}}, M\right]=e$.

Let $\bar{M}=\overline{\Gamma_{i_{1}}} \cdot \overline{\Gamma_{i_{2}}} \cdots \overline{\Gamma_{i_{m}}}$.

Since for each $j, \overline{\Gamma_{i_{j}}}=\overline{\Gamma_{i_{j}}^{x_{j}}}$ we get that

$$
f\left(\overline{\Gamma_{i_{j}}}, \bar{M}\right)=\left[\Gamma_{i_{j}}^{x_{j}}, M\right] / G_{3}=e_{G_{3}} .
$$

Theorem 4.2 Let $Q \in \mathcal{P}$ be an intersection point. Let $\Gamma(Q)=\left\{\Gamma_{i_{1}}, \ldots, \Gamma_{i_{m}}\right\}$ and $M=\Gamma_{i_{1}}^{x_{1}} \cdots \Gamma_{i_{m}}^{x_{m}}$. Then

$$
S(\bar{M})=\left\langle\overline{\Gamma(Q)} \cup\left(\bigcap_{\Gamma \in \Gamma(Q)} S(\bar{\Gamma})\right)\right\rangle .
$$

We call $S(\bar{M})$ the stabilizer of the intersection point $Q$.

Proof We start by proving that

$$
S(\bar{M}) \supseteq\left\langle\overline{\Gamma(Q)} \cup\left(\bigcap_{\Gamma \in \Gamma(Q)} S(\bar{\Gamma})\right)\right\rangle .
$$

$\overline{\Gamma(Q)} \subseteq S(\bar{M})$ : We have already shown that if $\Gamma \in \Gamma(Q)$, then $f(\bar{\Gamma}, \bar{M})=\bar{e}$ and hence $f(\bar{M}, \bar{\Gamma})=f((\bar{\Gamma}, \bar{M}))^{-1}=\bar{e}$. 
$\bigcap_{\Gamma \in \Gamma(Q)} S(\bar{\Gamma}) \subseteq S(\bar{M}):$ If $x \in \bigcap_{\Gamma \in \Gamma(Q)} S(\bar{\Gamma})$, then for any $\Gamma \in \Gamma(Q)$, we have $f(\bar{\Gamma}, x)=e$. This means that also the product $\prod_{\Gamma \in \Gamma(Q)} f(\bar{\Gamma}, x)=\bar{e}$, so by Lemma 4.1,

$$
\bar{e}=\prod_{\Gamma \in \Gamma(Q)} f(\bar{\Gamma}, x)=f\left(\left(\prod_{\Gamma \in \Gamma(Q)} \bar{\Gamma}\right), x\right)=f(\bar{M}, x) .
$$

Therefore $x \in S(\bar{M})$.

Since $S(\bar{M})$ is a subgroup and we have shown that it contains the union of $\overline{\Gamma(Q)}$ and $\bigcap_{\Gamma \in \Gamma(Q)} S(\bar{\Gamma})$, it clearly contains the subgroup generated by the union.

To complete the proof, we prove the opposite inclusion:

$$
S(\bar{M}) \subseteq\left\langle\overline{\Gamma(Q)} \cup\left(\bigcap_{\Gamma \in \Gamma(Q)} S(\bar{\Gamma})\right)\right\rangle .
$$

Let $\bar{x} \in S(\bar{M}) \subseteq G / G_{2}=\left\langle\overline{\Gamma(Q)}, \overline{\Gamma(Q)^{c}} \mid \bar{R}\right\rangle$. Then $\bar{x}$ can be written as $\bar{x}=\bar{z} \cdot \bar{y}$ where $\bar{z} \in\langle\overline{\Gamma(Q)}\rangle, \bar{y} \in\left\langle\Gamma(Q)^{c}\right\rangle$. We will prove $\bar{y} \in \bigcap_{\Gamma \in \Gamma(Q)} S(\bar{\Gamma})$.

Since $\overline{z^{-1}} \in\langle\overline{\Gamma(Q)}\rangle \subseteq S(\bar{M})$, so $\bar{y}=\overline{z^{-1}} \cdot \bar{z} \cdot \bar{y}=\overline{z^{-1}} \bar{x}$. Hence we get $\bar{y} \in S(\bar{M})$.

Let $l_{x}$ be a line passing through $Q$ and $\Gamma_{x}$ be the generator associated with it. Recall that $\bar{y} \in S(\bar{\Gamma})$ if $f(\bar{\Gamma}, \bar{y})=\bar{e}$. We need to prove that $f\left(\overline{\Gamma_{x}}, \bar{y}\right)=\bar{e}$ in $G_{2} / G_{3}$. From Remark 3.3 we know that $G_{2} / G_{3}$ is a direct sum of groups

$$
G_{2} / G_{3}=\bigoplus_{p \in \mathcal{P}} C_{p}
$$

so it remains to prove that $f\left(\left[\overline{\Gamma_{x}}, \bar{y}\right]\right)=\bar{e}$ in $C_{p}$, for all $p \in \mathcal{P}$. Let $p \in \mathcal{P}$. We have to show that the projection of the coset $\left[\overline{\Gamma_{x}}, \bar{y}\right] / G_{3}$ on $C_{p}$ is trivial.

We separate our proof into three cases.

Case $1 p=Q$.

Since $\bar{y} \in\left\langle\overline{\Gamma(Q)^{c}}\right\rangle=\left\langle\overline{\Gamma(p)^{c}}\right\rangle$ then the projection is trivial by the definition of $C_{p}$.

Case $2 l_{x}$ does not pass through $p$.

In this case, $\overline{\Gamma_{x}} \in\left\langle\overline{\Gamma(p)^{c}}\right\rangle$ and therefore the projection is trivial, by the definition of $C_{p}$.

Case $3 \quad p \neq Q$ and $l_{x}$ does pass through $p$.

By definition of $\mathrm{S}, f\left(\prod_{\Gamma \in \Gamma(Q)} \bar{\Gamma}, \bar{y}\right)=\bar{e}$. This means by the properties of $f$ (Lemma 4.1) that $\prod_{\Gamma \in \Gamma(Q)} f(\bar{\Gamma}, \bar{y})=\bar{e}$. Since $l_{x}$ passes through $p$, all the other lines passing 
through $Q$ do not cross $p$. So if we project any $f\left(\overline{\Gamma_{z}}, \bar{y}\right)$ where $\Gamma_{z}$ is any other generator from $\Gamma(Q)$, we get the identity. So in $C_{p}$,

$$
\bar{e}=\prod_{\Gamma \in \Gamma(Q)} f(\bar{\Gamma}, \bar{y})=f\left(\overline{\Gamma_{x}}, \bar{y}\right)
$$

and thus $f\left(\overline{\Gamma_{x}}, \bar{y}\right)=\bar{e}$.

\section{Fundamental groups which are semidirect product}

Theorem 5.1 Let $G=\left(\bigoplus_{i=1}^{n} A_{i}\right) \oplus \mathbb{Z}^{l}$ where $A_{i}$ are free groups and let $\psi: G \longrightarrow G$ be a projection where $\operatorname{im}(\psi)=\left\langle y_{1}, \ldots, y_{k}\right\rangle \cong \mathbb{F}_{k}$. Then, there exists $r, 1 \leq r \leq n$, such that for all $g \in G, \operatorname{pr}_{r}(g)=e$ implies that $\psi(g)=e$ and $\psi=\psi \circ \mathrm{pr}_{r}$, where $\mathrm{pr}_{r}: G \rightarrow G$ is the projection onto the subgroup of $G$ naturally isomorphic to $A_{r}$.

Proof Let us denote the $\mathbb{Z}^{l}$ component as $A_{0}$. Since $\psi$ is a projection, $\psi\left(y_{1}\right)=y_{1}$ and $\psi\left(y_{2}\right)=y_{2}$. Then $\psi\left(\left[y_{1}, y_{2}\right]\right)=\left[y_{1}, y_{2}\right] \neq e$. Note

$$
\left[y_{1}, y_{2}\right]=\bigoplus_{i=1}^{n} \operatorname{pr}_{i}\left(\left[y_{1}, y_{2}\right]\right)+\operatorname{pr}_{0}\left(\left[y_{1}, y_{2}\right]\right) \text {. }
$$

Since $A_{0}$ is abelian, $\operatorname{pr}_{0}\left(\left[y_{1}, y_{2}\right]\right)=e$, and therefore

$$
\left[y_{1}, y_{2}\right]=\bigoplus_{i=1}^{n} \operatorname{pr}_{i}\left(\left[y_{1}, y_{2}\right]\right) \text {. }
$$

Since $\psi$ is a homomorphism,

$$
\begin{aligned}
\psi\left(\left[y_{1}, y_{2}\right]\right) & =\psi\left(\operatorname{pr}_{1}\left(\left[y_{1}, y_{2}\right]\right) \oplus \cdots \oplus \operatorname{pr}_{n}\left(\left[y_{1}, y_{2}\right]\right)\right) \\
& =\psi\left(\operatorname{pr}_{1}\left(\left[y_{1}, y_{2}\right]\right)\right) \oplus \cdots \oplus \psi\left(\operatorname{pr}_{n}\left(\left[y_{1}, y_{2}\right]\right)\right) \neq e .
\end{aligned}
$$

This means that there is $r, 1 \leq r \leq n$, such that $\psi\left(\operatorname{pr}_{r}\left[y_{1}, y_{2}\right]\right) \neq e$, so if we denote $a:=\operatorname{pr}_{r}\left(y_{1}\right)$ and $b:=\operatorname{pr}_{r}\left(y_{2}\right)$ we get $[\psi(a), \psi(b)] \neq e$.

Since $a, b \in A_{r}$, if $x \in A_{j}$ where $j \neq r$, then $[x, a]=[x, b]=e$. We get that

$$
[\psi(x), \psi(a)]=[\psi(x), \psi(b)]=e .
$$

So $\psi(x)$ commutes with two noncommutative elements in a free group and hence $\psi(x)=e$, for all $x \in A_{j}, j \neq r$. This means that if $g \in G$ and $\operatorname{pr}_{r}(g)=e$, then $\psi(g)=e$. 
For each $g \in G, g=v_{1} \oplus \cdots \oplus v_{n} \oplus w, v_{j} \in A_{j}, w \in \mathbb{Z}^{l}$, we have that $\psi(g)=$ $\psi\left(v_{1} \oplus \cdots \oplus v_{n} \oplus w\right)=\psi\left(v_{1}\right) \oplus \cdots \oplus \psi\left(v_{n}\right) \oplus \psi(w)=\psi\left(v_{r}\right)=\psi\left(\operatorname{pr}_{r}(g)\right)$. Therefore $\psi=\psi \circ \operatorname{pr}_{r}$.

For proving the next result, we use the following theorem from Lyndon and Schupp [14]:

Theorem 5.2 Let $\phi$ be an epimorphism from a finitely generated free group $F$ to a free group $G$. Then $F$ has a basis $Z=Z_{1} \cup Z_{2}$ such that $\phi$ maps $\left\langle Z_{1}\right\rangle$ isomorphically onto $G$ and maps $\left\langle Z_{2}\right\rangle$ to $e$.

Corollary 5.3 Let $G=\left(\bigoplus_{i=1}^{n} A_{i}\right) \oplus \mathbb{Z}^{l}$ where $A_{i}$ are free groups and let $\psi: G \longrightarrow G$ be a projection where $\operatorname{im}(\psi)=\left\langle y_{1}, \ldots, y_{k}\right\rangle \cong \mathbb{F}_{k}$. For the same $r$ as in Theorem 5.1, we can find elements $\left\{z_{1}, \ldots, z_{m} \mid m \geq 0\right\} \subseteq A_{r}$ such that

$$
\left\{\operatorname{pr}_{r}\left(y_{1}\right), \ldots, \operatorname{pr}_{r}\left(y_{k}\right), z_{1}, \ldots, z_{m} \mid m \geq 0\right\}
$$

are generators of $A_{r}$ and $\psi\left(z_{i}\right)=e$.

Proof From Theorem 5.1 and the fact that $\psi$ is a projection $(\psi \circ \psi=\psi)$, we get that $\operatorname{pr}_{r} \circ \psi \circ \operatorname{pr}_{r} \circ \psi=\operatorname{pr}_{r} \circ \psi$. Therefore, $\operatorname{pr}_{r} \circ \psi$ is a projection.

Now since $\left(\operatorname{pr}_{r} \circ \psi\right)\left(A_{r}\right) \subseteq A_{r}$,

$$
\left.\left.\left(\operatorname{pr}_{r} \circ \psi\right)\right|_{A_{r}} \circ\left(\operatorname{pr}_{r} \circ \psi\right)\right|_{A_{r}}=\left.\left(\left(\operatorname{pr}_{r} \circ \psi\right) \circ\left(\mathrm{pr}_{r} \circ \psi\right)\right)\right|_{A_{r}}=\left.\left(\mathrm{pr}_{r} \circ \psi\right)\right|_{A_{r}} .
$$

By assumption,

$$
\operatorname{im}(\psi)=\left\langle y_{1}, \ldots, y_{k}\right\rangle \text { is a free group. }
$$

Now we claim $\left\{\operatorname{pr}_{r}\left(y_{1}\right), \ldots, \operatorname{pr}_{r}\left(y_{k}\right)\right\}$ are the generators of free group $\mathbb{F}_{k}$. If not, there is a nontrivial word $w=W\left(y_{1}, \ldots, y_{k}\right)$ so that $\operatorname{pr}_{\mathrm{r}}(w)=W\left(\operatorname{pr}_{r}\left(y_{1}\right), \ldots, \operatorname{pr}_{r}\left(y_{k}\right)\right)=e$. Therefore

$$
\left(\psi \circ \operatorname{pr}_{r}\right)(w)=\psi\left(\operatorname{pr}_{r}(w)\right)=\psi(e)=e .
$$

By Theorem 5.1, $\psi=\psi \circ \operatorname{pr}_{r}$, therefore $\psi(w)=\left(\psi \circ \operatorname{pr}_{r}\right)(w)=e$ which means that $w=e$, a contradiction to $(*)$.

In conclusion, we get that $\left.\left(\operatorname{pr}_{r} \circ \psi\right)\right|_{A_{r}}: A_{r} \rightarrow A_{r}$ is a projection such that $\operatorname{im}\left(\operatorname{pr}_{r} \circ \psi\right)=$ $\left\langle\operatorname{pr}_{r}\left(y_{1}\right), \ldots, \operatorname{pr}_{r}\left(y_{k}\right)\right\rangle \cong \mathbb{F}_{k}\left(\operatorname{pr}_{r}\left(y_{1}\right), \ldots, \operatorname{pr}_{r}\left(y_{k}\right)\right.$ are the generators of $\left.\mathbb{F}_{k}\right)$.

By Theorem 5.2, $A_{i}$ has a basis $B=B_{1} \cup B_{2}$ such that $\operatorname{im}\left(\operatorname{pr}_{r} \circ \psi\right)=\left\langle B_{1}\right\rangle$ and $\left(\operatorname{pr}_{r} \circ \psi\right)\left(B_{2}\right)=e$. Since $\operatorname{pr}_{r} \circ \psi$ is a projection, we can assume that $B_{1}=$ $\left\langle\operatorname{pr}_{r}\left(y_{1}\right), \ldots, \operatorname{pr}_{r}\left(y_{k}\right)\right\rangle$. Let $B_{2}=\left\{z_{1}, \ldots, z_{m} \mid \operatorname{pr}_{r} \circ \psi\left(z_{i}\right)=e\right\}$, which means that $\left(\psi \circ \operatorname{pr}_{r} \circ \psi\right)\left(z_{i}\right)=e$. As we proved earlier, $\psi \circ \operatorname{pr}_{r}=\psi$, so $(\psi \circ \psi)\left(z_{i}\right)=e$. But $\psi \circ \psi=\psi$, therefore $\psi\left(z_{i}\right)=e$. 
This implies that $A_{r}$ has generators

$$
\left\{\operatorname{pr}_{r}\left(y_{1}\right), \ldots, \operatorname{pr}_{r}\left(y_{k}\right), z_{1}, \ldots, z_{m}\right\}
$$

which satisfy the needed properties.

Without loss of generality, we temporarily change to a local numeration.

Let $G=\pi_{1}\left(\mathbb{C}^{2}-\Sigma\right)=\left\langle\Gamma_{1}, \ldots, \Gamma_{n}, \Gamma_{n+1}, \ldots, \Gamma_{l} \mid R\right\rangle$, where $\Gamma(A)=\left\{\Gamma_{1}, \ldots, \Gamma_{n}\right\}$ are the generators of the lines which participate in a specific multiple intersection point called $A$.

Definition 5.4 [12] Let $G$ be a group with a given presentation $G=\langle X \mid R\rangle$. A Tietze transformation $T_{i}(1 \leq i \leq 4)$ is a transformation of $\langle X \mid R\rangle$ to a presentation $G=\left\langle X^{\prime} \mid R^{\prime}\right\rangle$ of one of the following types:

$\left(\mathrm{T}_{1}\right)$ If $r$ is a word in $\langle X\rangle$ and $r=e$ is a relation that holds in $G$, then let $X^{\prime}=$ $X, R^{\prime}=R \cup\{r\}$.

$\left(\mathrm{T}_{2}\right)$ If $r \in R$ is such that the relation $r=e$ holds in the group $\langle X \mid R-r\rangle$, then let $X^{\prime}=X, R^{\prime}=R \backslash\{r\}$.

$\left(\mathrm{T}_{3}\right)$ If $w$ is a word in $\langle X\rangle$ and $z \notin X$, put $X^{\prime}=X \cup\{z\}, R^{\prime}=R \cup\left\{w z^{-1}\right\}$.

$\left(\mathrm{T}_{4}\right)$ If $z \in X$ and $w$ is a word in the other elements of $X$ such that $w z^{-1} \in R$, then substitute $w$ for $z$ in every other element of $R$ to get $\widetilde{R}$ and take $X^{\prime}=$ $X-\{z\}, R^{\prime}=\widetilde{R}$.

Before introducing the notion of a braid monodromy [16], we have to make some constructions. From now, we will work in $\mathbb{C}^{2}$. Let $E$ (resp. $D$ ) be a closed disk on $x$-axis (resp. $y$-axis), and let $C$ be a part of an algebraic curve in $\mathbb{C}^{2}$ located in $E \times D$. Let $\pi_{1}: E \times D \rightarrow E$ and $\pi_{2}: E \times D \rightarrow D$ be the canonical projections, and let $\pi=\left.\pi_{1}\right|_{C}: C \rightarrow E$. Assume $\pi$ is a proper map, and $\operatorname{deg} \pi=n$. Let $N=$ $\left\{x \in E \mid \# \pi^{-1}(x)<n\right\}$, and assume $N \cap \partial E=\varnothing$. Now choose $M \in \partial E$ and let $K=K(M)=\pi^{-1}(M)$. By the assumption that $\operatorname{deg} \pi=n \quad(\Rightarrow \# K=n)$, we can write $K=\left\{a_{1}, a_{2}, \ldots, a_{n}\right\}$. Under these constructions, from each loop in $E-N$, we can define a braid in $B_{n}[M \times D, K]$ in the following way:

(1) Since $\operatorname{deg} \pi=n$, we can lift any loop in $E-N$ with a base point $M$ to a system of $n$ paths in $(E-N) \times D$ which start and finish at $\left\{a_{1}, a_{2}, \ldots, a_{n}\right\}$.

(2) Project this system into $D$ (by $\pi_{2}$ ), to get $n$ paths in $D$ which start and end at the image of $K$ in $D$ (under $\pi_{2}$ ). These paths actually form a motion.

(3) Induce a braid from this motion; see Garber [8]. 
To conclude, we can match a braid to each loop. So we get a map $\alpha: \pi_{1}(E-N, M) \rightarrow$ $B_{n}[M \times D, K]$, which is also a group homomorphism. This map is called the braid monodromy of $C$ with respect to $E \times D, \pi_{1}, M[16 ; 8]$.

The following remark demonstrates the necessity of the condition that there are no parallel lines in the affine plane:

Remark 5.5 Let $\Sigma$ be a line arrangement with no parallel line in the affine plane. Let $\Gamma_{1}, \ldots, \Gamma_{l}$ be the generators of $\pi_{1}\left(\mathbb{C}^{2}-\Sigma\right)$. Then

$$
\Gamma_{1} \cdots \Gamma_{l} \in Z\left(\pi_{1}\left(\mathbb{C}^{2}-\Sigma\right)\right) .
$$

Proof Let $\alpha: \pi_{1}(\mathbb{C}-\Sigma) \rightarrow B_{n}$ be the braid monodromy of $\Sigma$. By Remark 4.7 in [3], the closed braid determined by the product $\alpha\left(\Gamma_{1}\right) \cdots \alpha\left(\Gamma_{l}\right)$ is actually a link of the curve at infinity. In a generic curve, we have $\alpha\left(\Gamma_{1}\right) \cdots \alpha\left(\Gamma_{l}\right)=\Delta^{2}$, where $\Delta$ is the braid which rotates by $180^{\circ}$ counterclockwise all the strands together. One of the presentations of the fundamental group is $\left\langle\Gamma_{1}, \ldots, \Gamma_{l} \mid \alpha\left(s_{i}\right)\left(\Gamma_{j}\right)=\Gamma_{j}, \forall i, \forall j\right\rangle$, where $s_{i}$ is the loop created in the $x$-axis as a result of the projection of the line arrangement on the plane [13]. Therefore, $\alpha\left(s_{i}\right)$ is a braid acting on the generators of the fundamental group. As a result $\alpha\left(s_{1} \cdots s_{n}\right)\left(\Gamma_{j}\right)=\Gamma_{j}$, for all $j$, which means $\Delta^{2}\left(\Gamma_{j}\right)=\Gamma_{j}$, for all $j$. It is known that $\Delta^{2}(x)=x^{\Gamma_{1} \cdots \Gamma_{l}}$ and thus $\Gamma_{j}^{\Gamma_{1} \cdots \Gamma_{l}}=\Gamma_{j}$. Hence, $\Gamma_{1} \cdots \Gamma_{l} \in Z\left(\pi_{1}\left(\mathbb{C}^{2}-\Sigma\right)\right)$.

Let $G=\pi_{1}\left(\mathbb{C}^{2}-\Sigma\right)=\left\langle\Gamma_{1}, \ldots \Gamma_{n}, \Gamma_{n+1}, \ldots, \Gamma_{l} \mid R\right\rangle$, where $\Gamma(A)=\left\{\Gamma_{1}, \ldots, \Gamma_{n}\right\}$ are the generators of the lines which pass through in a specific multiple intersection point called $A$.

Let us recall that the lines passing through $Q$ are $\left\{L_{i_{1}}, \ldots, L_{i_{m}}\right\}$. Recall also $M=$ $\Gamma_{i_{1}}^{x_{1}} \Gamma_{i_{2}}^{x_{2}} \cdots \Gamma_{i_{m}}^{x_{m}}$

Theorem 5.6 With the same assumptions of Theorem 5.1 and Remark 5.5, let $H:=$ $\left\langle\Gamma_{i_{1}}, \ldots, \Gamma_{i_{m-1}}\right\rangle$. Let $N$ be the normal closure of $\left\{\Gamma(Q)^{c}, M\right\}$. Let $f_{1}: H \rightarrow G$ be the natural embedding and $f_{2}: G \rightarrow G / N$ the natural homomorphism. Then:

(1) $G / N \cong \mathbb{F}_{m-1}$ which is generated by $f_{2}\left(\Gamma_{i_{j}}\right), 1 \leq j \leq m-1$.

(2) $H \cong \mathbb{F}_{m-1}$.

(3) $G=N \rtimes \mathbb{F}_{m-1}$.

(4) There exists a projection $h: G \rightarrow G \quad\left(h^{2}=h\right)$, such that $\operatorname{im}(h)=H$ and $\operatorname{ker}(h)=N$. 
Proof (1) We can present $G / N=\left\langle\Gamma(Q), \Gamma(Q)^{c} \mid R, M, \Gamma(Q)^{c}\right\rangle$. Denote $\Gamma(Q)^{c}=$ $\left\{z_{1}, \ldots, z_{k}\right\}$. By applying iteratively Tietze's transformation $\left(T_{4}\right)$ for every $z_{i} \in$ $\Gamma(Q)^{c}$, we obtain $G / N=\langle\Gamma(Q) \mid \widehat{R}, \widehat{M}\rangle$, where $\widehat{R}, \widehat{M}$ are obtained from $R, M$ by substituting $e$ for $z_{i}, 1 \leq i \leq k$, in every other element of $R$ and $M$, respectively.

Let $v \in \mathcal{P}$ with $v \neq Q$ be an intersection point, and let $r$ be the relations induced by $v$. Now we can get the following:

(a) If there is a line $L$ that goes through both points $v$ and $Q$ and the generator attached to $L$ is $\Gamma_{i_{j}}$ for some $j, 1 \leq j \leq m$, then the relations $r$ become $\left[\Gamma_{i_{j}}^{x}, 1, \ldots, 1\right]$ for some $x$. These relations are trivial.

(b) If the points $v$ and $Q$ do not share any line, then the relations $r$ become $[1, \ldots, 1]$, which are also trivial.

Let us denote by $\widetilde{W}$ the word $W$ after rewriting. Due to the above observations, using the presentation $G / N=\langle\Gamma(Q) \mid \widetilde{R}, \widetilde{M}\rangle$, then

$$
G / N=\left\langle\Gamma_{i_{1}}, \ldots, \Gamma_{i_{m}} \mid\left[\Gamma_{i_{1}}^{\widetilde{x_{i_{1}}}}, \ldots, \Gamma_{i_{m}}^{\widetilde{x_{i_{m}}}}\right], \widetilde{M}\right\rangle .
$$

This is equal to

$$
\left\langle\Gamma_{i_{1}}, \ldots, \Gamma_{i_{m}} \mid\left[\Gamma_{i_{1}}^{\widetilde{x_{i_{1}}}}, \widetilde{M}\right], \ldots,\left[\Gamma_{i_{m}}^{\widetilde{x_{i_{m}}}}, \widetilde{M}\right], \widetilde{M}\right\rangle=\mathbb{F}_{m-1} .
$$

(2) Assume otherwise. Then there exists a nontrivial word $w\left(\Gamma_{i_{1}}, \ldots, \Gamma_{i_{m-1}}\right)=e$. So applying $f_{2}$, we get a nontrivial word $w\left(f_{2}\left(\Gamma_{i_{1}}\right), \ldots, f_{2}\left(\Gamma_{i_{m-1}}\right)\right)$ which is not possible by (1).

(3) From the first paragraph, we get that $f_{2} \circ f_{1}$ is a surjective function from $\mathbb{F}_{m-1}$ to $\mathbb{F}_{m-1}$ and therefore it is an isomorphism.

(4) Derived directly from (2) and (3).

By the last theorem: Let $Q \in \mathcal{P}, \Gamma(Q)=\left\{\Gamma_{i_{1}}, \ldots, \Gamma_{i_{m}}\right\}$. Then there is a projection $h: G \rightarrow G$ such that $\operatorname{im}(h)=\left\langle\Gamma_{i_{1}}, \ldots, \Gamma_{i_{m}}\right\rangle$ and $\operatorname{ker}(h)$ is the normal closure of $\left\langle\Gamma(Q)^{c}, M\right\rangle$.

By Theorem 5.1 and Corollary 5.3 we get that there is $r, 1 \leq r \leq n$, such that $A_{r}=\left\{w_{1}, \ldots, w_{n-1}, z_{1}, \ldots, z_{k} \mid k \geq 0\right\}$ and $h=h \circ \mathrm{pr}_{r}, \mathrm{pr}_{r} \circ h$ is a projection, $\Gamma_{i}=h\left(w_{i}\right), h\left(z_{i}\right)=e, b_{i}=\Gamma_{i} w_{i}^{-1}, h\left(b_{i}\right)=e$ (note that $h$ has the role of $\left.\psi\right)$.

Claim 5.7 $\overline{A_{r}}=\operatorname{pr}_{\bar{r}}(S(\bar{M}))$. 
Proof We start by proving that $\operatorname{pr}_{r}(M)=e$ : for all $x, y$ and $j_{1} \neq j_{2}$. Since $\Gamma_{i_{j_{1}}}$ and $\Gamma_{i_{j_{2}}}$ are different generators of the free group $\operatorname{im}(h), h\left(\left[\Gamma_{i_{j_{1}}}^{x} \Gamma_{i_{j_{2}}}^{y}\right]\right) \neq e$. We know that $h=h \circ \operatorname{pr}_{r}$, so we get

$$
\left(h \circ \operatorname{pr}_{r}\right)\left(\left[\Gamma_{i_{j_{1}}}^{x} \Gamma_{i_{j_{2}}}^{y}\right]\right)=h\left(\left[\Gamma_{i_{j_{1}}}^{x} \Gamma_{i_{j_{2}}}^{y}\right]\right) \neq e,
$$

so $\operatorname{pr}_{r}\left(\left[\Gamma_{i_{j_{1}}}^{x} \Gamma_{i_{j_{2}}}^{y}\right]\right) \neq e$. Hence, for $x=x_{j_{1}}, y=x_{j_{2}}, \operatorname{pr}_{r}\left(\left[\Gamma_{i_{j_{1}}}^{x_{j_{1}}}, \Gamma_{i_{j_{2}}}^{\left.x_{j_{2}}\right]}\right) \neq e\right.$. We know that $\left[\Gamma_{i_{1}}^{x_{j_{1}}}, M\right]=e$, so the elements $\operatorname{pr}_{r}\left(\Gamma_{i_{j}}^{x_{j}}\right), j=1,2$, commute with $M$ but do not commute with each other in the free group $A_{r}$, therefore $\operatorname{pr}_{r}(M)=e$.

The next step is to prove that $\overline{A_{r}} \subseteq S(\bar{M})$. Let $a \in \overline{A_{r}}$. Then there exists $a^{*} \in A_{r}$ such that $\overline{a^{*}}=a$. Since $\operatorname{pr}_{r}(M)=e$ and $a^{*} \in A_{r}$, by the properties of direct sum $\left[a^{*}, M\right]=e$. Therefore $\left[a^{*}, M\right] / G_{3}=e_{G_{3}}$. By definition $f(a, \bar{M})=e_{G_{3}}$ which implies that $a \in S(\bar{M})$.

Hence, we have $\overline{A_{r}} \subseteq \operatorname{pr}_{\bar{r}}(S(\bar{M}))$. The opposite inclusion is trivial by definition, and thus $\overline{A_{r}}=\operatorname{pr}_{\bar{r}}(S(\bar{M}))$.

By Theorem 4.2, $S(\bar{M})=\left\langle\left\{\overline{\Gamma_{i_{1}}}, \ldots, \overline{\Gamma_{i_{m}}}\right\} \cup\left\{\bigcap_{k=1}^{m} S\left(\overline{\Gamma_{i_{k}}}\right)\right\}\right\rangle$, therefore

$$
\begin{aligned}
\overline{A_{r}} & =\operatorname{pr}_{\bar{r}}\left(\left\langle\left\{\Gamma_{i_{1}}, \ldots, \Gamma_{i_{m}}\right\} \cup \bigcap_{k=1}^{m} S\left(\Gamma_{i_{k}}\right)\right\rangle\right) \\
& =\left\langle\left\{\operatorname{pr}_{\bar{r}}\left(\Gamma_{i_{1}}\right), \ldots, \operatorname{pr}_{\bar{r}}\left(\Gamma_{i_{m}}\right)\right\} \cup \operatorname{pr}_{\bar{r}}\left(\bigcap_{k=1}^{m} S\left(\overline{\Gamma_{i_{k}}}\right)\right)\right\rangle .
\end{aligned}
$$

We claim that the right part of this generating set is trivial:

Claim 5.8 $\operatorname{pr}_{\bar{r}}\left(\bigcap_{k=1}^{m} S\left(\Gamma_{i_{k}}\right)\right)=\{e\}$.

Proof It is known that for any two sets $A, B$ and a function $F$ that $F(A \cap B) \subseteq$ $F(A) \cap F(B)$. Therefore $\operatorname{pr}_{\bar{r}}\left(\bigcap_{k=1}^{m} S\left(\Gamma_{i_{k}}\right)\right) \subseteq \bigcap_{k=1}^{m}\left(\operatorname{pr}_{\bar{r}}\left(S\left(\Gamma_{i_{k}}\right)\right)\right)$.

As we mentioned $A_{r}=\left\langle\operatorname{pr}_{r}\left(\Gamma_{1}\right), \ldots, \operatorname{pr}_{r}\left(\Gamma_{m}\right), z_{1}, \ldots, z_{q} \mid q \geq 0\right\rangle$.

In other words, $A_{r}=\left\langle\beta_{1}, \ldots, \beta_{m+q}\right\rangle$ where

$$
\beta_{k}= \begin{cases}\operatorname{pr}_{r}\left(\Gamma_{i_{k}}\right) & k \leqslant m, \\ z_{k-m} & m+1 \leqslant k \leqslant m+q .\end{cases}
$$

Let $\bar{g} \in \operatorname{pr}_{\bar{r}}\left(S\left(\Gamma_{i_{k}}\right)\right) \subseteq \overline{A_{r}}$, therefore $\bar{g}=\overline{\beta_{1}{ }^{t_{1}}} \cdots \overline{\beta_{q+m}{ }^{t_{m+q}}}$. Without loss of generality, we can choose that $g=\beta_{1}^{t_{1}} \cdots \beta_{m+q}^{t_{m+q}}$. By Theorem 5.1 we know that

$$
G=A_{1} \oplus \cdots \oplus A_{r} \oplus \cdots \oplus A_{n} \oplus \mathbb{Z}^{\ell} .
$$


We define $D=A_{1} \oplus \cdots \oplus \widehat{A_{r}} \oplus \cdots \oplus A_{n} \oplus \mathbb{Z}^{\ell}$, then $G=A_{r} \oplus D$.

Hence, there exists $g_{2} \in D$ such that $\bar{g} \oplus \overline{g_{2}} \in S\left(\overline{\Gamma_{i_{k}}}\right)$.

Let $\varphi: G \rightarrow D$ be the natural projection, and define $\eta_{k}:=\varphi\left(\Gamma_{i_{k}}\right)$. Hence, it implies that $\Gamma_{i_{k}}=\operatorname{pr}_{r}\left(\Gamma_{i_{k}}\right) \oplus \eta_{k}$.

$$
\begin{aligned}
e_{G_{3}} & =f\left(\overline{\Gamma_{i_{k}}}, \overline{g \oplus g_{2}}\right)=f\left(\overline{\operatorname{pr}_{r}\left(\Gamma_{i_{k}}\right) \oplus \eta_{k}}, \overline{g \oplus g_{2}}\right) \\
& =f\left(\overline{\operatorname{pr}_{r}\left(\Gamma_{i_{k}}\right)}, \bar{g}\right) f\left(\overline{\operatorname{pr}_{r}\left(\Gamma_{i_{k}}\right)}, \overline{g_{2}}\right) f\left(\overline{\eta_{k}}, \bar{g}\right) f\left(\overline{\eta_{k}}, \overline{g_{2}}\right) .
\end{aligned}
$$

By the definition of $f$, this implies that

$$
\left[\overline{\operatorname{pr}_{r}\left(\Gamma_{i_{k}}\right)}, \bar{g}\right]\left[\overline{\operatorname{pr}_{r}\left(\Gamma_{i_{k}}\right)}, \overline{g_{2}}\right]\left[\overline{\eta_{k}}, \bar{g}\right]\left[\overline{\eta_{k}}, \overline{g_{2}}\right] \in G_{3} .
$$

Since $\operatorname{pr}_{r}\left(\Gamma_{i_{k}}\right), g \in A_{r}$, and $g_{2}, \eta_{k} \in D$, then $\left[\operatorname{pr}_{r}\left(\Gamma_{i_{k}}\right), g_{2}\right]=\left[\eta_{k}, g\right]=e_{G_{3}}$. Therefore $\left[\operatorname{pr}_{r}\left(\Gamma_{i_{k}}\right), g\right]\left[\eta_{k}, g_{2}\right] \in G_{3}=\left(A_{r}\right)_{3} \oplus D_{3}$, which means $\left[\operatorname{pr}_{r}\left(\Gamma_{i_{k}}\right), g\right] \in\left(A_{r}\right)_{3}$ and $\left[\eta_{k}, g_{2}\right] \in D_{3}$.

The fact that $\left(A_{r}\right)_{2} /\left(A_{r}\right)_{3}=\left\langle\left[\beta_{i}, \beta_{j}\right] \mid 1 \leq i<j \leq m+q\right\rangle$ is derived directly from the definition of $A_{r}$. Hence, $\left(A_{r}\right)_{3} \ni\left[\operatorname{pr}_{r}\left(\Gamma_{i_{k}}\right), g\right]=\left[\beta_{k}, g\right]=\left[\beta_{k}, \beta_{1}^{t_{1}} \cdots \beta_{m+q}^{t_{m+q}}\right]=$ $\left[\beta_{k}, \beta_{1}\right]^{t_{1}} \cdots\left[\beta_{k}, \beta_{k}\right]^{t_{k}} \cdots\left[\beta_{k}, \beta_{m+q}\right]^{t_{m+q}}$.

Note that $\left[\beta_{k}, \beta_{k}\right]=e$. By [10], $\left\{\left[\beta_{k}, \beta_{j}\right] \mid j \neq k\right\}$ are generators (or inverses) of $\left(A_{r}\right)_{2} /\left(A_{r}\right)_{3}$ which is a free abelian group. Since every generator appears at most once, we get $t_{j}=0$ for all $j \neq k$.

In conclusion, $\bar{g}=\overline{\beta_{k}^{t_{k}}} \in\left\langle\overline{\beta_{k}}\right\rangle=\left\langle\overline{\operatorname{pr}_{r}\left(\Gamma_{i_{k}}\right)}\right\rangle$. So $\bar{g} \in\left\langle\overline{\operatorname{pr}_{\bar{r}}\left(\Gamma_{i_{k}}\right)}\right\rangle$.

To summarize, we have shown that if $\bar{g} \in\left\langle\operatorname{pr}_{\bar{r}}\left(S\left(\Gamma_{i_{k}}\right)\right)\right\rangle$ then $\bar{g} \in\left\langle\operatorname{pr}_{\bar{r}}\left(\Gamma_{i_{k}}\right)\right\rangle$. Therefore, $\left\langle\operatorname{pr}_{\bar{r}}\left(S\left(\Gamma_{i_{k}}\right)\right)\right\rangle \subseteq\left\langle\operatorname{pr}_{\bar{r}}\left(\Gamma_{i_{k}}\right)\right\rangle$.

In conclusion, $\bigcap_{k=1}^{m}\left\langle\operatorname{pr}_{\bar{r}}\left(S\left(\Gamma_{i_{k}}\right)\right)\right\rangle \subseteq \bigcap_{k=1}^{m}\left\langle\operatorname{pr}_{\bar{r}}\left(\Gamma_{i_{k}}\right)\right\rangle=e$, so $\operatorname{pr}_{\bar{r}}\left(\bigcap_{k=1}^{m} S\left(\Gamma_{i_{k}}\right)\right)=$ $\{e\}$ as claimed.

Since $\Gamma_{i_{1}} \cdots \Gamma_{i_{m}}=e, \overline{A_{r}}=\left\langle\overline{\Gamma_{i_{1}}}, \ldots, \overline{\Gamma_{i_{m-1}}}\right\rangle$. As a result, $\overline{A_{r}}=\operatorname{pr}_{\bar{r}}\left\langle\Gamma_{i_{1}}, \ldots, \Gamma_{i_{m-1}}\right\rangle$. Consequently, $\operatorname{rank}\left(A_{r}\right) \leq m-1$.

We know that $A_{r}=\left\langle w_{1}, \ldots, w_{m-1}, z_{1}, \ldots, z_{t} \mid t \geq 0\right\rangle$. Combining these two facts together and by Theorem 5.2, we get $A_{r}=\left\langle w_{1}, \ldots, w_{m-1}\right\rangle$, where $y_{i}=\left(\operatorname{pr}_{r} \circ h\right)\left(\Gamma_{i_{j}}^{x_{j}}\right)$.

From Theorem 5.1, $h \circ \mathrm{pr}_{r}=h$. Since $h$ is a projection $h^{2}=h$. Hence, we get

$$
\operatorname{pr}_{r} \circ h \circ \operatorname{pr}_{r} \circ h=\operatorname{pr}_{r} \circ h \circ h=\operatorname{pr}_{r} \circ h,
$$

which means that $\operatorname{pr}_{r} \circ h$ is a projection too. Recall that

$$
A_{r}=\left\langle\left(\mathrm{pr}_{r} \circ h\right)\left(\Gamma_{i_{1}}\right), \ldots,\left(\mathrm{pr}_{r} \circ h\right)\left(\Gamma_{i_{m-1}}\right)\right\rangle .
$$


Therefore $\left(\operatorname{pr}_{r} \circ h\right)\left(w_{j}\right)=\left(\operatorname{pr}_{r} \circ h\right) \circ\left(\operatorname{pr}_{r} \circ h\right)\left(\Gamma_{i_{j}}\right)=\left(\operatorname{pr}_{r} \circ h\right)\left(\Gamma_{i_{j}}\right)=w_{j}$. Hence we get that $\left(\operatorname{pr}_{r} \circ h\right)\left(A_{r}\right)=A_{r}$.

Now if $l_{i}$ is a line that does not pass through the point $Q$ and $\Gamma_{i}$ is the generator of $l_{i}$, then $h\left(\Gamma_{i}\right)=e$ and therefore $\left(\operatorname{pr}_{r} \circ h\right)\left(\Gamma_{i}\right)=\operatorname{pr}_{r}(e)=e$.

From this investigation, we get the following theorem.

Theorem 5.9 Let $\Sigma$ be a line arrangement and $\pi_{1}\left(\mathbb{C}^{2}-\Sigma\right) \simeq\left(\bigoplus_{i=1}^{n} A_{i}\right) \oplus \mathbb{Z}^{l}$ where $A_{i}$ is a free group. Then for any multiple point $Q$ in the arrangement with $k$ lines, namely, $\left\{l_{1}, \ldots, l_{k}\right\}$, there exists $r, 1 \leq r \leq n$, and a projection onto $A_{r}, \varphi_{Q}: G \rightarrow G$ such that $A_{r}=\left\langle\varphi_{Q}\left(\Gamma_{1}\right), \ldots, \varphi_{Q}\left(\Gamma_{k}\right)\right\rangle \cong \mathbb{F}_{k-1}$. If $l_{j}$ is a line which does not pass through the point, then $\varphi_{Q}\left(\Gamma_{j}\right)=e$.

Moreover, if $p_{1}, \ldots, p_{m}$ are the multiple points of $\Sigma$ and $n_{i}$ is the number of lines pass through the point $p_{i}$, then $G \cong\left(\bigoplus_{i=1}^{m} C_{i}\right) \oplus B$, where $C_{i} \cong \mathbb{F}_{n_{i}-1}$. If $l$ is a line which does not pass through $p_{i}$ and let $\Gamma$ be its corresponding generator, then $\operatorname{pr}_{i}(\Gamma)=e\left(\right.$ where $\operatorname{pr}_{i}$ is the projection onto $\left.C_{i}\right)$.

\section{Main theorems}

Theorem 6.1 Let $\Sigma \subseteq \mathbb{C}^{2}$ be a line arrangement which has no pair of parallel lines. Then if

$$
\pi_{1}\left(\mathbb{C}^{2}-\Sigma\right)=\bigoplus_{i=1}^{r} A_{i} \oplus \mathbb{Z}^{l},
$$

where $A_{i}$ are free groups. Then $\beta(\Sigma)=0$.

Proof Assume by negation that $\beta(\Sigma) \neq 0$ which means that there is at least one cycle in the graph of $\Sigma$. Let us pick a minimal cycle in the following sense. The cycle contains $r$ points, namely $\left\{p_{1}, \ldots, p_{r}\right\}$ and $p_{i}$ is connected in the cycle only to $p_{i-1}$ and $p_{i+1}$ (the indices are taken modulo $r$ ). In other words, a cycle with no subcycles.

By Theorem 5.9, we can write $G=\left(\bigoplus_{i=1}^{r} C_{i}\right) \oplus B_{1}$ where $B_{1}$ is not necessarily abelian. If $l$ is a line that does not pass through the points in the cycle and let $\Gamma$ be its corresponding generator, then $\operatorname{pr}_{i}(\Gamma)=e, 1 \leq i \leq r$. Define $N=$ $\left\langle\Gamma_{X_{1}}, \ldots, \Gamma_{X_{t}}, \Gamma_{1} \cdots \Gamma_{n}\right\rangle$, where $\Gamma_{X_{1}}, \ldots, \Gamma_{X_{t}}$ are the generators of lines that do not participate in an intersection point $p_{i}, 1 \leq i \leq r$. Denote $Z=\Gamma_{1} \cdots \Gamma_{n}$. Let $H:=G / N$.

Let $n_{i}$ be the number of lines passing through $p_{i}$. 
On one hand, since we have $b:=\sum_{i=1}^{r}\left(n_{i}-1\right)$ lines participating in the cycle, then if we denote by $\Gamma_{1}, \ldots, \Gamma_{b}$ the generators associated with the lines participating in the cycle, then $H \cong\left\langle\Gamma_{1}, \ldots, \Gamma_{b} \mid \widetilde{R}, \widetilde{Z}\right\rangle$ where $\widetilde{R}$ is the relations which are derived from the original relations. Therefore, it is easy to see that $\operatorname{rank}(\bar{H}) \leq b-1$.

On the other hand, for $1 \leq i \leq r$ and $1 \leq j \leq t, \operatorname{pr}_{i}\left(\Gamma_{X_{j}}\right)=e$ and also $\Gamma_{1} \cdots \Gamma_{n} \in Z(G)$ so $\operatorname{pr}_{i}\left(\Gamma_{1} \cdots \Gamma_{n}\right)=e$. Thus $\operatorname{pr}_{i}(N)=e$ and hence $C_{i} / N \cong C_{i}$. This implies that $H=G / N \cong\left(\bigoplus_{i=1}^{r} C_{i} \oplus B_{1}\right) / N$. Since $C_{i} \cong \mathbb{F}_{n_{i}-1}$ and $\overline{C_{i}} \cong \mathbb{Z}^{n_{i}-1}$, we have $\bar{H}=$ $\left(\bigoplus_{i=1}^{r} \overline{C_{i}}\right) \oplus \overline{B_{1} / N}$ which implies that $\bar{H}=\left(\bigoplus_{i=1}^{r} \mathbb{Z}^{n_{i}-1}\right) \oplus \overline{B_{1} / N} \cong \mathbb{Z}^{b} \oplus \overline{B_{1} / N}$. Thus $\operatorname{rank}(\bar{H}) \geq b$, a contradiction.

We are now ready to prove the converse of Fan's theorem.

Theorem 6.2 Let $\Sigma \subseteq \mathbb{C P}^{2}$ be a line arrangement. If

$$
\pi_{1}\left(\mathbb{C P}^{2}-\Sigma\right)=\bigoplus_{i=1}^{r} A_{i} \oplus \mathbb{Z}^{l},
$$

where $A_{i}$ are free groups, then $\beta(\Sigma)=0$.

Proof Suppose that $\Sigma \subset \mathbb{C P}^{2}$ is an arrangement of complex projective lines such that $\pi_{1}\left(\mathbb{C P}^{2} \backslash \Sigma\right)$ is isomorphic to a direct product of free groups. Let $L_{0} \subset \mathbb{C P}^{2}$ be a complex projective line which is in general position to $\Sigma$. Then $L_{0}$ and $\Sigma$ intersect at double points. Choose an arbitrary complex projective line of $\Sigma$ and denote this line by $L_{1}$. Note that $\mathbb{C}^{2} \cong \mathbb{C P}^{2} \backslash L_{0} \cong \mathbb{C P}^{2} \backslash L_{1}$. By applying the product theorem of Oka and Sakamoto [17], we have

$$
\begin{aligned}
\pi_{1}\left(\mathbb{C P}^{2} \backslash\left(\Sigma \cup L_{0}\right)\right) & =\pi_{1}\left(\left(\mathbb{C P}^{2} \backslash L_{1}\right) \backslash\left(\left(\Sigma \backslash L_{1}\right) \cup L_{0}\right)\right) \\
& \cong \pi_{1}\left(\left(\mathbb{C P}^{2} \backslash L_{1}\right) \backslash\left(\Sigma \backslash L_{1}\right)\right) \oplus \pi_{1}\left(\left(\mathbb{C P}^{2} \backslash L_{1}\right) \backslash L_{0}\right) \\
& \cong \pi_{1}\left(\mathbb{C P}^{2} \backslash \Sigma\right) \oplus \mathbb{Z} .
\end{aligned}
$$

This calculation shows that $\pi_{1}\left(\mathbb{C P}^{2} \backslash\left(\Sigma \cup L_{0}\right)\right)$ is a product of free group. Note that

$$
\pi_{1}\left(\mathbb{C P}^{2} \backslash\left(\Sigma \cup L_{0}\right)\right)=\pi_{1}\left(\left(\mathbb{C P}^{2} \backslash L_{0}\right) \backslash\left(\Sigma \backslash L_{0}\right)\right) .
$$

Note that $\Sigma \backslash L_{0}$ is a union of complex lines in the complex plane $\mathbb{C P}^{2} \backslash L_{0}$ such that no two lines of this arrangement are parallel.

By Theorem 6.1 $\beta\left(\Sigma \backslash L_{0}\right)=0$, therefore $\beta(\Sigma)=\beta\left(\Sigma \backslash L_{0}\right)=0$. 
Acknowledgements We wish to thank David Garber for the experience he shared with us and the time he invested in improving the paper. We are extremely grateful for his great efforts in order to make this paper worthy.

We would also like to thank the anonymous referee for his fruitful advice. In an earlier version submitted to the journal, the paper was written under the implicit assumption that no two lines of the arrangement are parallel to each other. Under this assumption we give the proof of Theorem 6.1. The referee suggested us to make this assumption explicit. The proof of Theorem 6.2 is given by the referee in earlier referee report of this paper.

\section{References}

[1] W A Arvola, The fundamental group of the complement of an arrangement of complex hyperplanes, Topology 31 (1992) 757-765 MR1191377

[2] A D R Choudary, A Dimca, Ş Papadima, Some analogs of Zariski's theorem on nodal line arrangements, Algebr. Geom. Topol. 5 (2005) 691-711 MR2153112

[3] D C Cohen, A I Suciu, The braid monodromy of plane algebraic curves and hyperplane arrangements, Comment. Math. Helv. 72 (1997) 285-315 MR1470093

[4] M Falk, Homotopy types of line arrangements, Invent. Math. 111 (1993) 139-150 MR1193601

[5] K-M Fan, On parallel lines and free groups arXiv:0905.1178

[6] KM Fan, Position of singularities and the fundamental group of the complement of a union of lines, Proc. Amer. Math. Soc. 124 (1996) 3299-3303 MR1343691

[7] K-M Fan, Direct product of free groups as the fundamental group of the complement of a union of lines, Michigan Math. J. 44 (1997) 283-291 MR1460414

[8] D Garber, On the fundamental groups of complements of plane curves, $\mathrm{PhD}$ thesis, Bar-Ilan University (2001)

[9] D Garber, M Teicher, $\mathbf{U}$ Vishne, $\pi_{1}$-classification of real arrangements with up to eight lines, Topology 42 (2003) 265-289 MR1928653

[10] M Hall, Jr, The theory of groups, second edition, Chelsea Publishing Co., New York (1976) MR0414669 Reprint of the 1968 edition

[11] T Jiang, SS-T Yau, Diffeomorphic types of the complements of arrangements of hyperplanes, Compositio Math. 92 (1994) 133-155 MR1283226

[12] D L Johnson, Presentations of groups, London Math. Soc. Lecture Notes Ser. 22, Cambridge Univ. Press (1976) MR0396763

[13] E R V Kampen, On the fundamental group of an algebraic curve, Amer. J. Math. 55 (1933) 255-267 MR1506962 
[14] R C Lyndon, P E Schupp, Combinatorial group theory, Ergebnisse der Math. und ihrer Grenzgebiete 89, Springer, Berlin (1977) MR0577064

[15] W Magnus, A Karrass, D Solitar, Combinatorial group theory.Presentations of groups in terms of generators and relations, revised edition, Dover, New York (1976) MR0422434

[16] B Moishezon, M Teicher, Braid group technique in complex geometry. I. Line arrangements in $\mathbf{C P}^{2}$, from: "Braids (Santa Cruz, CA, 1986)", (J S Birman, A Libgober, editors), Contemp. Math. 78, Amer. Math. Soc. (1988) 425-555 MR975093

[17] M Oka, K Sakamoto, Product theorem of the fundamental group of a reducible curve, J. Math. Soc. Japan 30 (1978) 599-602 MR513072

[18] P Orlik, H Terao, Arrangements of hyperplanes, Grund. der Math. Wissenschaften 300, Springer, Berlin (1992) MR1217488

[19] R Randell, The fundamental group of the complement of a union of complex hyperplanes, Invent. Math. 69 (1982) 103-108 MR671654

[20] S Wang, S S-T Yau, Rigidity of differentiable structure for new class of line arrangements, Comm. Anal. Geom. 13 (2005) 1057-1075 MR2216152

Department of Mathematics, Bar Ilan University, 52900 Ramat Gan, Israel

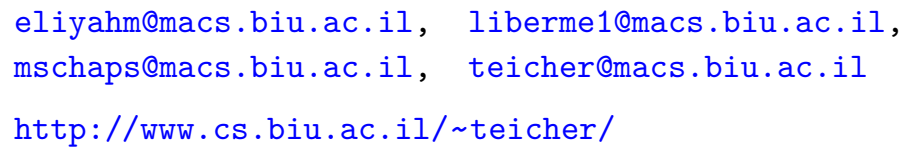

Received: 26 February 2009 Revised: 14 October 2009 